\title{
Effects of Obstructions on the Accuracy of Bluetooth Contact Tracing
}

\author{
David Labrique ${ }^{1}$ \\ dlabrique@gmail.com \\ ${ }^{1}$ Independent Researcher, Towson, MD, USA
}

\begin{abstract}
During the COVID-19 pandemic, the idea of facilitating contact tracing using Bluetooth is becoming widespread due to the prevalence of smartphones. The automatic classification of encounters between smartphones is challenging due to variations in signal strength - resulting in high false positive and false negative rates. For example, obstructions between two smartphones can reduce the strength of received signals and thus increase the calculated distance. This can benefit contact tracing by preventing a contact from being added to the database when people are on opposite side of a wall, but can also harm tracing efforts if two people are close together but their bodies reduce the signal between devices. A Raspberry Pi Bluetooth emitter and a phone receiver were placed 1 meter apart, and various obstacles were placed between them to simulate normal obstacles. Drywall and stud walls were shown to be ineffective at reducing Bluetooth signal strength. Cinder block, and especially the human body, were found to effectively lower Bluetooth strength so that the distance estimate was higher. The results of these experiments imply Bluetooth contact tracing will involve many false positives and negatives.
\end{abstract}

Keywords-Bluetooth, Contact Tracing, RSSI, Obstructions, Covid-19

\section{INTRODUCTION}

\section{A. Project Description}

This project aims to determine the reduction of Bluetooth signal strength through various common materials, including through humans. This is relevant to PiPact's goals of improving Bluetooth contact tracing as reduction or lack of reduction of Bluetooth signals across barriers can lead to false negatives and false positives in contact tracing.

\section{B. Background Information}

To contain pandemics, an anonymous, widely distributable system of automated contact tracing is very helpful. Contact tracing is an effective strategy to contain infectious disease epidemics, such as the ongoing Covid-19 global pandemic that has to date cost over 600,000 human lives. This epidemiologic approach involves identifying all persons who have come into contact with a positive case of the infection under investigation. The sooner that exposed contacts are found, and placed under quarantine, the lower the risk of continued spread of disease through a population. The manual process of contact tracing is time consuming, especially in determining which contacts came into sufficient proximity of the case for an adequate length of time to classify as an exposure. As such, digital strategies to assist human contact tracers are being pursued. Current contact tracing efforts being scaled up around the globe usually involve using Bluetooth apps on smartphones, which send out and detect Bluetooth pings at regular intervals. Because these pings contain the transmitter's power level (Tx), smartphones are able to compare the received signal strength indicator (RSSI) to the Tx to calculate distance; if the distance is less than a certain value (less than 2 meters for Covid-19), the hashed, or encrypted, data is then sent to a database. When a patient with a contact tracing app tests positive for a disease and notifies the system, everyone whose phones detected proximity to the patient in the last few weeks is notified to get tested and to self-quarantine [7].

Obstructing materials can diminish the received RSSI of a Bluetooth signal, creating the miscalculation of a greater distance than the actual distance between two persons. This miscalculation can benefit contact tracing, by preventing false positives when two people are, for instance, only one meter apart but separated by a wall, as is often the case in apartment buildings. In large urban areas where people live in high-rise buildings, the risk of misclassification of neighbors living beside or below an infection person could lead to a high number of false positives.

It can also harm contact tracing efforts by creating false negatives where people are actually close together but the phones are obstructed by their bodies; for example, if two people are facing each other with phones in their back pockets the signal may be attenuated. Water, which humans are primarily composed of, is a strong obstructer of Bluetooth signals [2], as shown by the diminishing of wireless performance in high humidity environments [1]. The Raspberry Pi is a small computer chip with a $-56 \mathrm{dBm}$ (decibel-milliwatts) of Tx when configured as a low energy iBeacon. Android Beacon Scanner is an open-source application that can identify and measure the Tx and RSSI of iBeacons and perform distance estimations based on those measurements [3].

\section{HYPOTHESIS/HYPOTHESES}

The first hypothesis, $\mathrm{H} 1_{\mathrm{A}}$, is that if common wall materials are placed between a Bluetooth emitter and a receiver, the signal strength detected by the receiver will remain strong enough compared to one expected to be received at a 1 meter unobstructed distance, thus generating false positives in a Bluetooth contact tracing proximity detection program. The null hypothesis, $\mathrm{H}_{0}$, is that the signal 
strength will be sufficiently occluded by common wall materials to prevent those false positives. The second hypothesis, $\mathrm{H} 2 \mathrm{~A}$, is that occlusion of signal strength by the human body will be sufficient to create false negatives in a Bluetooth contact tracing proximity detection program via decreasing observed signal strength. This type of error will lead to the software interpreting the signal as being adequately distanced, even though the persons are standing close together, in an environment with no obstructions such as a wall or divider. The second null hypothesis, $\mathrm{H} 20$, is that the human body does not significantly occlude Bluetooth transmission.

\section{EXPERIMENTS AND DATA COLLECTIONS}

\section{A. Plan and Execution}

One Raspberry Pi was put in nonconnectable undirected low energy advertising mode via command lines [8], and placed at one end of a 1 meter long wooden board, set up as a testing platform, with the midpoint demarcated for consistency across experiments. At the other end of the board a Samsung Galaxy Note 8 phone with the open source software "Beacon Scanner" installed and running [3] was placed, with the scan rate set to measure signal strength every 5 seconds. Each experiment lasted for one minute, with the RSSI value of the Pi's signal recorded at each time interval.

TABLE I. EXAMPLE EXPERIMENT OVERVIEW (TABLE HEAD)

\begin{tabular}{|l|l|l|l|}
\hline Exp.\# & \multicolumn{1}{|c|}{ Hypothesis } & \multicolumn{1}{|c|}{ Reason } & \multicolumn{1}{|c|}{ Obstruction } \\
\hline 1 & N/A & Control & None \\
\hline 2 & H1 & $\begin{array}{l}\text { Common type of } \\
\text { wall }\end{array}$ & 1 1 1', drywall \\
\hline 3 & H1 & $\begin{array}{l}\text { Common type of } \\
\text { wall }\end{array}$ & 2x 1', drywall \\
\hline 4 & H1 & $\begin{array}{l}\text { Common type of } \\
\text { wall }\end{array}$ & $\begin{array}{l}\text { Stud wall } \\
\text { simulacrum (2 1' } \\
\text { drywalls nailed to } \\
2.5 \text { inch pinewood } \\
2 \times 4 \text { board) }\end{array}$ \\
\hline 5 & H1 & $\begin{array}{l}\text { Common type of } \\
\text { wall }\end{array}$ & 18 ' cinderblock \\
\hline 6 & H2 & $\begin{array}{l}\text { Simulate phone } \\
\text { in back } \\
\text { pocket/third } \\
\text { person between } \\
\text { those involved in } \\
\text { tracing }\end{array}$ & $\begin{array}{l}\text { Perpendicular } \\
\text { human }\end{array}$ \\
\hline 7 & H1 + H2 & $\begin{array}{l}\text { Simulate phone } \\
\text { in back pocket of } \\
\text { human against } \\
\text { wall }\end{array}$ & $\begin{array}{l}\text { Perpendicular } \\
\text { human and stud } \\
\text { wall simulacrum }\end{array}$ \\
\hline
\end{tabular}

After each experiment, the scanner was disabled and its final estimated distance recorded before the app's Bluetooth $\log$ data was reset. The list of obstructions tested are described in Table 1, above. Two experiments were conducted with one and then two sheets of drywall, each half an inch thick, placed at the midpoint of the test board. One experiment was conducted using a stud wall simulacrum, consisting of two $1 / 2$ inch drywalls nailed to a 2.5 inch wide piece of pinewood at the midpoint. Another experiment was done with an 8" thick cinder block at the midpoint. An experiment where a human test subject laid across the board, perpendicular to it at its midpoint, was conducted. An experiment where a human laid on the board perpendicular to it at its midpoint with the stud wall simulacrum between the human and the receiver was also carried out. See attached images for a visual depiction of the experiments.

\section{B. Data Relevance}

The "control" test, where there was no obstruction between the $\mathrm{Pi}$ and the receiver phone, was done to determine a baseline Tx of the Pi's RSSI at one meter, and to test the accuracy of Beacon Scanner's distance estimation algorithm. Common types of wall materials were then placed at the midpoint to simulate the interference possible to a contact tracing application being used on opposite sides of a wall. A human laid down at the midpoint to simulate the effect of a phone held in the user's back pocket or a third person standing between two contact tracing app users, obstructing the signal and, of course, the level of exposure. A human at the midpoint together with the simulacrum stud wall simulates scenarios such as two people sitting on opposite sides of a wall at a restaurant.

Figure 1

Illustration of real-life scenarios being simulated by experiments 2-7 as described in Table 1, at left.

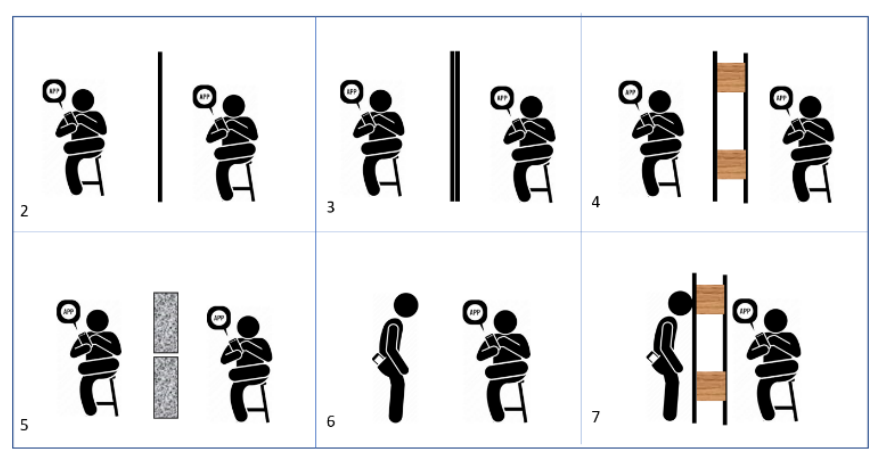

\section{Examples}

Figures 2 and 3 display the data, as captured over the course of these experiments.

Figure 2

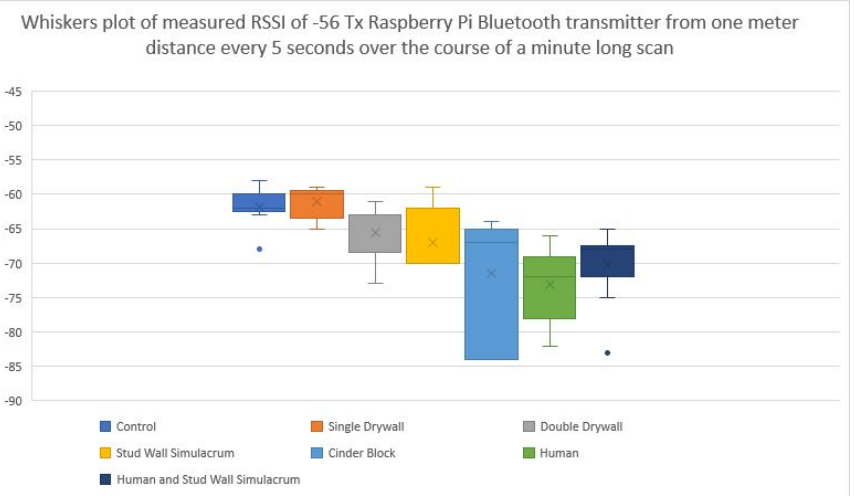


Table II.

$X^{2}$ test results of RSSI values across experiments using the control as the expected distribution, showing that only Exp 5-7 were significantly different $(\mathrm{p}<0.05$, italics $)$ from the control.

\begin{tabular}{|l|c|}
\hline & $\begin{array}{l}X^{2} \text { Test } \\
\text { Results }\end{array}$ \\
\hline Control & 0.999 \\
\hline Single Drywall & 0.985 \\
\hline Double Drywall & 0.576 \\
\hline Stud Wall Simulacrum & 0.003 \\
\hline Cinder Block & 0.001 \\
\hline Human & 0.043 \\
\hline $\begin{array}{l}\text { Human and Stud Wall } \\
\text { Simulacrum }\end{array}$ & \\
\hline
\end{tabular}

Figure 3

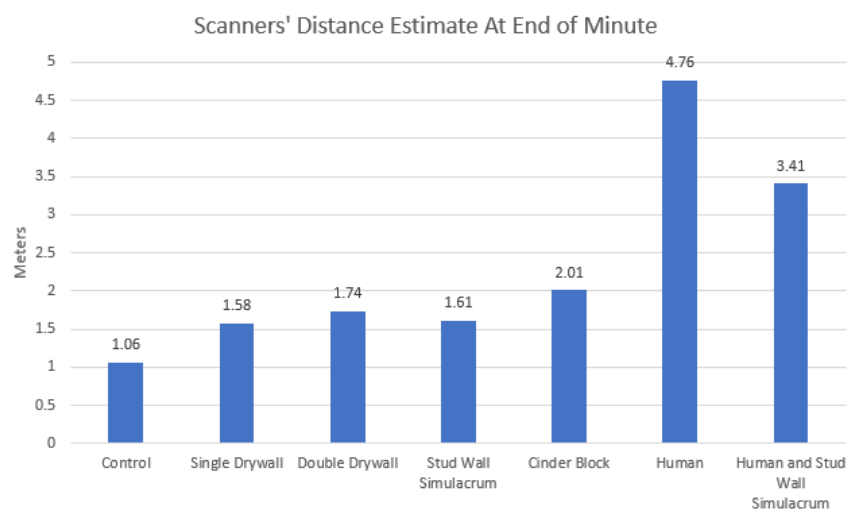

IV. ANALYSIS AND AlgORITHMS

\section{A. Description}

There was substantial variation in the RSSI measured during each experiment, and overlap between the RSSI ranges of each experiment. Box and whisker charts were generated using Microsoft Excel to compare the distribution of the data. The mean is shown as an $\mathrm{X}$ and the median as a line through the box. The Beacon Scanner's distance estimations after 1 minute were also recorded and plotted using a bar graph. A chi squared test was used to evaluate how different each RSSI distribution was from the control's distribution.

\section{B. Results and Examples}

The control had the lowest interquartile range of any experiment, and the highest first quartile RSSI, as expected. The single drywall did not significantly decrease the RSSI, as although the $4^{\text {th }}$ quartile and $1^{\text {st }}$ quartile were lower than in the control, the mean and second quartile were higher. The double drywall was the first test where there was a clear decrease from the control, as all but the $1^{\text {st }}$ quartile were below the $4^{\text {th }}$ quartile of control. The results for the stud wall simulacrum were similar. The cinder block had the greatest range in RSSI values in the experiment, with its $1^{\text {st }}$ quartile below the $4^{\text {th }}$ quartile of control and its $4^{\text {th }}$ quartile the lowest of any experiment at $-84 \mathrm{dBm}$. With a human, the mean and median and first quartile were below those of and other experiments. With both a human and a stud wall simulacrum, the median, mean, and quartiles were all higher than those of just a human, but its outlier is lower than any value for just a human. As shown by the $X^{2}$ test results displayed in Table II, using the control group RSSI distribution as the reference, the RSSI of Bluetooth through single and double drywall is not statistically significantly different from the control, the RSSI with a stud wall obstruction is mildly statistically significantly different than the control, and the RSSI measured during the cinder block, human, and human with stud wall were clearly statistically significantly different from the control.

\section{CONCLUSIONS}

\section{A. Hypothesis Evaluation}

$\mathrm{H} 1_{\mathrm{A}}$ was partially confirmed and partially refuted: thinner or less dense walls, such as 1 or 2 inches of drywall, or even a stud wall, do not reliably reduce the strength of a Bluetooth signal below that expected of a unobstructed signal at 1 meter (transmission risk) distance. As shown in Figure 3, the Beacon Scanner algorithm did not identify any of the drywall-type barriers as increasing the estimated distance between transmitter and receiver above 2 meters (the currently advised "safe distance" for coronavirus) [5], thus demonstrating the risk of possible false positives when individuals are separated by walls such as these. With a thicker, denser wall, such as cinder block, Bluetooth RSSI is statistically significantly reduced in signal (Table II), and the Beacon Scanner app falsely estimates a distance of 2 meters at an actual test distance of only 1 meter. In this case, the cinder block encounter would serendipitously be classified as 'safe', and not counted as an exposure event - as the two persons are separated by a cement wall. $\mathrm{H} 2 \mathrm{~A}_{\mathrm{A}}$ was confirmed as a human decreased RSSI to far below that of the control, also statistically significant, with the estimated distance as high as 4.76 meters, showing how a false negative would arise as the Bluetooth contact tracing app would classify the encounter between devices as being a safe distance.

\section{B. Noteworthy Conclusions}

A human body is highly effective at reducing the strength of a Bluetooth signal that passes through it. Existing Bluetooth applications are capable of accurately estimating distance using RSSI when there are no obstructions. Pure drywall and stud walls are about equally effective at blocking Bluetooth, but only enough to increase the distance estimation by a factor of 1.5 to 2 . Of the measured materials only the cinder block was capable of increasing the distance estimation to over 2 meters.

\section{General Lessons Learned}

Covid-19 can be spread through normal exhaled breath, speaking, shouting or singing [4], and people usually stand face to face while talking, often with phones in their back pockets. This study illustrates that human bodies 
drastically reduce Bluetooth signals, and thus risk confounding apps which are trying to automatically detect close-proximity encounters between individuals.

Furthermore, the fact that drywall, a commonly used building material in much of the United States, does not adequately diminish the Bluetooth signal is likely to lead to high numbers of false positives, especially in dense urban settings - like New York City, where rapid contact tracing would have been extremely useful given the serious nature of the pandemic in that population.

A different approach is therefore needed for accurate contact tracing using digital technology. Smart watches might be possible candidates for more accurate contact tracing as they are less obstructed by the body when worn on the wrist. Even under controlled conditions (no movement, constant humidity), RSSI can fluctuate wildly in a matter of seconds (so people passing right by each other may not register as having been close). Single measurements are likely to provide highly inaccurate distance and RSSI measurements. As infectious encounters usually require a longer exposure (10-15 minutes), one might consider capturing a larger number of RSSI values to accurately extract a distribution and median value of the signal. However, this risks draining battery life.

\section{NeXT STEPS}

Ultrasound has been touted by some as being more accurate than Bluetooth. NOVID, an organization testing this technology claims that ultrasound cannot pass through walls, preventing false positives, and a study has shown it is unaffected by being in pockets or by ambient music [6], though a test of the effect of wind and urban sources of ultrasound remains to be done. A field test of Bluetooth and ultrasound contact tracing using humans or robot dummies in a mock town would be more effective than this experiment in gauging the accuracy of contact tracing in environments with many obstructions. 